\title{
Question and Answer Sessions with Dr. Paulus T.V.M. de Jong
}

\section{Tokyo}

\section{Chairman: Dr. Yoshihisa Oguchi}

(Department of Ophthalmology, School of Medicine, Keio University)

Dr. Oguchi: Thank you very much, Dr. de Jong. It was a very interesting and instructive lecture. Dr. de Jong discussed the Rotterdam Study on AMD (age-related macular disease). I am surprised that the No. 1 factor in blindness among Caucasians is AMD, and that diseases differ from race to race. Now I would like to invite questions and comments from the audience.

Dr. Osamu Katsumi (Tokyo): Thank you, Professor de Jong. I have two questions. One regards the incidence. Does it differ according to locations, i.e. does it matter whether you live in Rotterdam or in Florida? Are there any differences in qualities of sunlight? Also, I was very surprised to see the results of hyperopia as a risk factor. What if myopia is more like $6 \mathrm{dpt}$ ?

Dr. de Jong: Your first question was if the incidence in Caucasians differs. We compared our data with those from the Beaver Dam Study in the USA and with the Blue Mountain Study in Australia. Incidences were slightly lower in the Rotterdam Study, but it was not a big difference. We also had a slightly lower prevalence in Rotterdam. This might be either due to small differences in examination techniques or to environmental factors. At this moment we are analyzing the data from the EUREYE Study. This study examines about 4,000 respondents from 8 different countries in Europe from Norway to Greece. Generally speaking, AMD prevalence data seem the same all over Europe. I cannot give definite figures yet, but we think that there is not really a big difference there despite differences in light exposure.

As to your questions about myopia ... Myopia is of course a problem while defining AMD for two reasons. If we have a neovascular disciform reaction in the macular area of a -8 or $-10 \mathrm{dpt}$ myopic eye, we are not sure if that is due to myopic Fuchs degeneration or due to AMD. In general, we say we need to see drusen before we call it AMD. AMD is a diagnosis by exclusion. So when you have an aspecific scar in the macular area without clear drusen, we can neither rule out AMD nor for example juvenile macular degeneration or presumed ocular histoplasmosis. Myopic fundi, at least in Caucasians, are less pigmented than hyperopic eyes, especially when you have a large field of choroidal atrophy and white myopic degenerations. You cannot see the drusen very well anymore. This may make it more difficult to detect drusen. Each diopter shift towards hyperopia gave a $6 \%$ higher rate of AMD. So, we think it is not due to confounding factors or to missing a few correct diagnoses. Asians have more myopia than Caucasians with its known complications, but a good thing seems to be that this leads to less AMD.

Dr. Oguchi: Dr. Katsumi asked two questions. The first question was if there is any relationship between AMD incidence and location. Dr. de Jong related that he took part in prevalence studies in 8 locations in Europe, but there was no obvious difference in prevalence among locations. The second was about hypermetropia. Honestly speaking, it is a first time for me to hear that a higher level of hypermetropia causes more AMD. As there are more

\begin{tabular}{ll}
\hline KARGER & ( ) 2004 S. Karger AG, Basel \\
0030-3755/04/2187-0039\$21.00/0 \\
$\begin{array}{l}\text { Fax +4161306 12 34 } \\
\begin{array}{l}\text { E-Mail karger@karger.ch } \\
\text { www.karger.com }\end{array}\end{array}$ & $\begin{array}{l}\text { Accessible online at: } \\
\text { www.karger.com/oph }\end{array}$
\end{tabular}


farsighted people in Europe than in Japan, there may be more AMD cases in Europe than in Japan. More questions from the audience?

Dr. Yutaka Imamura (Keio University): Thank you very much for your wonderful lecture. In Japan, more males, 3 times more males than females, are likely to show AMD development while the data from Dr. de Jong show more females with prevalent AMD. Would you explain this, please? I guess that more Japanese males than females smoke.

Dr. de Jong: My first remark regards how you found out that Japanese males have more AMD than females. Are these clinic-based data or population-based data? If you are talking about clinic-based data, they can be confounding because, for example, perhaps the male goes to the doctor earlier than the female or the other way around. I am not sure if it is common in Japan, but I know that in parts of China or in India the male is considered more important than the female, so they send boys to the doctor earlier than girls. So this may lead to bias. Before we accept that males have triple the AMD rate, I would like to know exactly where you got the data from. I must say, however, that in the pooled data from the Beaver Dam, Blue Mountains and Rotterdam Studies there was no longer any difference in AMD prevalence between males and females.

Dr. Imamura: Thank you. How about smokers in Holland? Are males more likely to smoke than females?

Dr. de Jong: It seems there are more smokers in Japan than in Holland. So is there here a gene protecting against smoking effects?

Holland has another problem. Older males smoke less. Females smoke more and more. So, we see a rising incidence of lung cancer in women. Our main concern is that the highschool population smokes a lot. It is so difficult to teach these youngsters not to smoke. I have no exact data why people start smoking, but I know the bad side effects for the women. I also have read that the type of lung cancer in women is even worse than in men because it starts more often multilocally and in the lung periphery than in the major bronchi.

Dr. Yoshiko Matsuhashi (Nagoya City): I would like to ask you one more question. Which causes the higher percentage of AMD, hyperopia, myopia or presbyopia?

Dr. de Jong: Hyperopia is a much higher risk factor than myopia. In all our age strata, myopia was protective against AMD. I am not aware of any report on the association between presbyopia and AMD after correcting for age.

Dr. Oguchi: Thank you very much, Dr. de Jong.

\section{Osaka}

\section{Chairman: Prof. Akira Negi}

(Department of Ophthalmology, Kobe University)

Dr. Negi: Thank you Dr. de Jong for your splendid lecture. Let me invite questions from the audience.

Dr. Yasuaki Kuwayama (Osaka Kouseinenkin Hospital): I have two questions. First, I would like to ask whether drusen in AMD are pathological or they simply represent an ageing phenomenon. While your lecture suggested that drusen are early indications of AMD, some believe that drusen are an ageing phenomenon. Do you think drusen by themselves constitute the pathology of earlystage AMD?

Next, you mentioned the study in the USA, in which nutritional supplements prevented patients from developing AMD. Do you think patients with drusen should be instructed to follow well-balanced nutrition? Does such an intervention reduce the risk of disease onset?

Dr. de Jong: In my view, drusen are a mixture of ageing phenomenon and pathology when we see the clear rise in prevalence with ageing. I also consider drusen one manifestation of malfunctioning retinal pigment epithelium (RPE). If it were pure ageing I had expected more people over the age of 75-80 years to have many or larger drusen. We have different hypotheses why drusen lead to AMD, for example antioxidant deficiency, inflammation, or vascular growth factor upregulation. Some groups have been giving more attention to the type of drusen. Professor Chakravarthy from Belfast (Queen's University and Royal Group of Hospitals) looked at about 200 patients with stage 4 AMD. She had a picture from many years earlier of these cases. People with many drusen developed more occult neovascular AMD, people with few drusen more classic neovascular AMD. So we now think that drusen are a manifestation of a dysfunctioning RPE. And the more drusen you have, the more sick your RPE is. We looked at many types and different clinical assessments of drusen and could not find correlations with the type of late AMD. However, the larger the area covered by drusen in the macula, the higher the chance of late AMD, but we still consider drusen to be a hallmark of AMD at all ages.

Dr. Kuwayama: I understood, but as you know, some doctors think that drusen are only an ageing phenomenon and not something particular to the disease. Do you think drusen represent very-early-stage AMD?

Dr. de Jong: Most people about the age of 70-75 have a few tiny drusen (on histology 10-20 years earlier), but we also found people who had no drusen on ophthalmoscopy.
40

Ophthalmologica 2004;218(suppl 1):39-41
Question and Answer Sessions with Dr. Paulus T.V.M. de Jong 
So I don't think drusen are necessarily present in every person, and I consider drusen, especially $>125 \mu \mathrm{m}$, to represent early AMD and to be a risk factor for late AMD. AMD is multifactorial, a combination of genetics and environment. We calculated that about 27\% of AMD is due to a genetic contribution and the remainder is due to the environment, e.g. food, drinking, smoking and other factors.

Dr. Kuwayama: So the answer to the first question is 'yes', right? My second question is that if we find drusen in a patient, should we always recommend good nutrition?

Dr. de Jong: With regard to the AREDS Study with nutritional supplements I am convinced after seeing our data on antioxidant intake in the diet that a well-balanced diet with vitamin $\mathrm{C}$ and $\mathrm{E}, \beta$-carotene and zinc protects against AMD. I think that would be a good suggestion. People having a diet with above median antioxidant intake in the Rotterdam Study had less AMD. So if I have a patient with stage 2 AMD or higher, I recommend taking antioxidants, but not in such high doses as in the AREDS Study, 5 times more than the recommended daily dose. I would like everyone with large drusen to change dietary habits and eat more green vegetables. Also zinc seems especially important. I'm not sure if rice contains much zinc, but fish, poultry, meat, dairy products and brown bread do. So I would recommend taking them.

Dr. Yozo Miyake (Nagoya University): Let me ask an additional question. I noticed the report of a very largescale supplementation study in the archives dating back a few years ago. The study examined categories $1-4$. While the data showed no effect of supplementation in categories 1 and 2, some benefits were observed in relatively advanced categories 3 and 4. I'm much interested in knowing whether or not the current evaluation system as mentioned by Dr. Kuwayama is valid for the evaluation of early-stage AMD. As Dr. de Jong pointed out in the lecture, the use of autofluorescence and other methods may facilitate a more accurate assessment of early-stage severity of disease.

When we look at the risk factors for AMD, we find there are several vascular factors, such as hypertension and arteriosclerosis. May I ask your opinion as to whether diabetics have more AMD?

Dr. de Jong: I'm sorry, I'm not sure if I caught all your questions. The AREDS Study showed antioxidant protection for the late stages. We now also for the early stages. My answer to the last question whether people with diabetes mellitus have more AMD is that we did not find this association in the Rotterdam Study. One explanation might be survival bias because people with severe diabetes have a shorter life expectancy and thus die before they can develop AMD. We looked on the other hand at the life expectancies of AMD, glaucoma and cataract, and when we adjusted for all confounders, we found no higher mortality in people with these diseases. So my feeling is that diabetes is not a major risk factor for AMD. Does that answer your question?

Dr. Negi: Recent studies showed that the incidence of polypoidal choroidal vasculopathy (PCV) is very high in Japan. I wonder how the situation in Japan can be compared with that in western countries. In Japan, polypoidal vasculopathy accounts for $30-40 \%$ of neovascular lesions.

Dr. de Jong: We can only correctly diagnose polypoidal disease when you have angiograms and preferably indocyanine green ones. In the Rotterdam Study, we made no angiograms, and thus it is hard to identify PCV. We can only guess from color fundus photographs for evidence of PCV. As far as I hear from incidental clinic-based reports, PCV is much more common in Japan than in Europe. I would very much like to see fundus pictures made in Japanese epidemiological AMD studies to compare them with ours.

Dr. Negi: The population in the Rotterdam Study included a substantial number of black people. I think the prevalence among black people may be somewhat lower than the prevalence in our data. I think their lifestyle is similar to the western style. Do the initial diagnostic findings of AMD in black people resemble those seen in Caucasians, or do they present more with AMD associated with PCV?

Dr. de Jong: I am not quite sure if African Americans have more PCV than whites. I think that African Americans have the same prevalence of early AMD, but they have less late-stage AMD (stage 4). I do not know of any studies comparing dietary intake or other lifestyle factors. I know for example that in a study, African blacks and Aborigines in Australia had less AMD. But I cannot compare the lifestyles or even the evidence for the higher frequency of polypoidal disease in blacks than in Caucasians. By the way, in the Rotterdam Study 99\% were of Caucasian origin.

Dr. Negi: Thank you very much. Regarding epidemiological studies, Japanese researchers have recently concluded a study on glaucoma and obtained very accurate data for the first time in Japan. The results were considerably different from those of western studies. As we understand from this experience, epidemiological studies are important as the bases for a scientific approach. Thank you very much again, Dr. de Jong. 